\title{
A new proposal of effective stress and critical distance for fatigue at notches
}

\author{
C. Vallellano, A. Navarro and V. Chaves \\ Department of Mechanical and Manufacturing Engineering, Universidad de Sevilla, Camino de los \\ Descubrimientos s/n. 41092 Sevilla, Spain
}

\begin{abstract}
The Theory of Critical Distance (TCD) and its associated methods have proved useful for predicting fatigue and fracture failure under stress gradients. This work reports the first analytical deduction of the effective stress and its critical distance in notched components under fatigue. The results, consistent with their LEFM counterparts, indicate that the most accurate representation of the Notch Effective Stress (NES) is the geometric mean of the stresses used in the Point and Line Methods respectively, evaluated at the critical distance of the material proposed by Taylor.
\end{abstract}

\section{Motivation}

The methods based on the Theory of Critical Distance (TCD) have proved highly useful engineering tools for analysing fatigue strength in notched components or elements under a stress gradient. Briefly, these methods assume fatigue failure in a component to occur when the effective stress at a given critical distance from the surface reaches a reference value such as the fatigue limit for the material. Taylor and co-workers reviewed the classical notions and models based on TDC, and demonstrated their ability to predict failure by effect of various phenomena including fatigue, fragile fracture or ductile fracture in components under stress gradients such as those resulting from notches, stress raisers or fretting fatigue in various types of materials (e.g. metals, polymers, ceramics, biological substrates) under both uniaxial and multiaxial loads [1-3]. However, effective stresses used so far, e.g. in the Line Method (LM) and Point Method (PM), and their critical distances, have only been checked mathematically for the stress gradient in cracks, but never for notches or more general gradients. As admitted by Prof. Taylor himself in the opening session of the European Conference on Fracture of 2006, these are essentially the strengths and weaknesses of TDC:

\footnotetext{
"In conclusion, the Theory of Critical Distances is a theory which has not received as much attention as it deserves. One barrier to its general acceptance is the lack of a clear theoretical basis to explain its success. This is a challenge for future work; however, we should remember that the first and most important test of any scientific theory is its ability to predict the experimental data: this is a test which the TCD passes with flying colours."
}

This work reports the first analytical deduction of the effective stress and its associated critical distance in a notched component under fatigue.

This is an Open Access article distributed under the terms of the Creative Commons Attribution License 4.0, which permits unrestricted use, distribution, and reproduction in any medium, provided the original work is properly cited. 


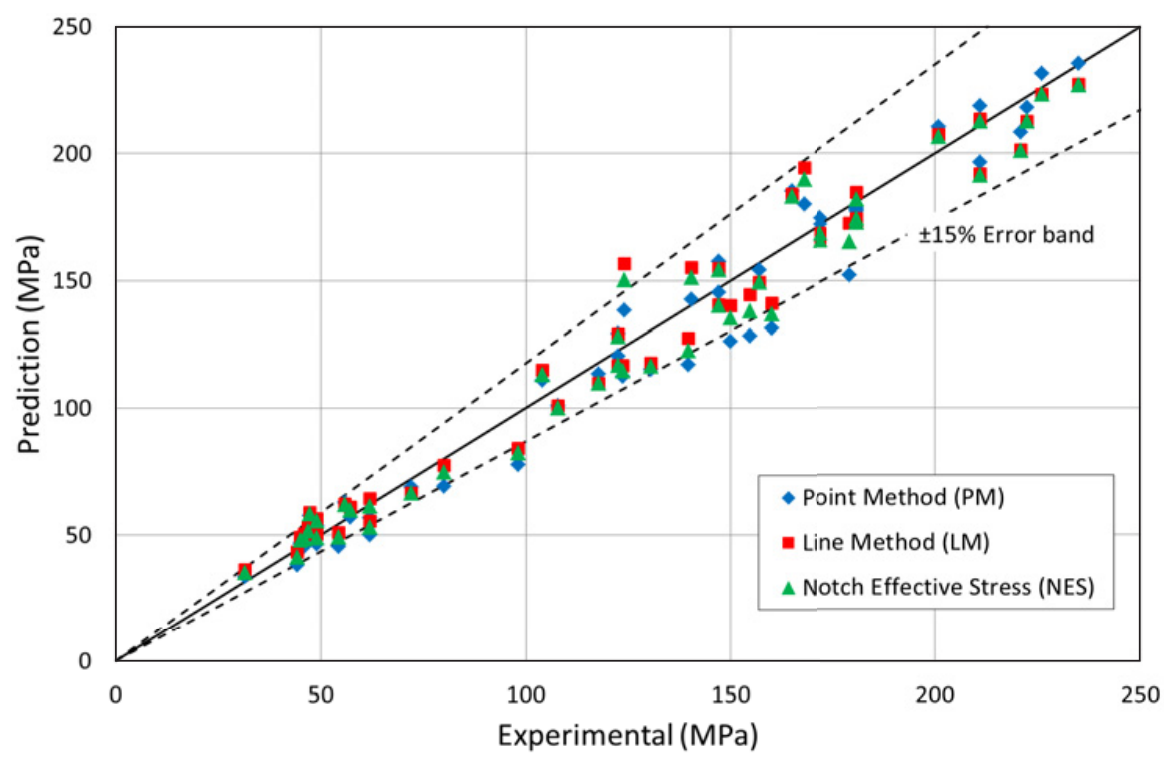

Figure 1. Predictions using the current NES versus the classical PM and LM approaches.

\section{Notch effective stress. Application}

Using conformal mapping and distributed dislocation techniques $[4,5]$, the SIF for a crack of length $d$ in the bottom of a notch can be expressed as the geometric mean of the mean stress $\left[\bar{T}_{z}^{0}(d)\right]$ and local stress $\left[T_{z}^{0}(d)\right]$ in the absence of cracks, calculated at a distance $d$, and multiplied by $\sqrt{\pi d}$. By using the critical distance of the material for fatigue failure, $L=\frac{1}{\pi}\left(K_{t h} / \sigma_{0}\right)^{2}$ (where $\sigma_{0}$ is now the fatigue limit of the material), a Notch Effective Stress (NES) can be defined,

$$
\sigma_{\text {eff }}(d)=\sqrt{\bar{T}_{z}^{0}(d) T_{z}^{0}(d)}
$$

Thus, the fatigue failure in a notched component will occur when the NES equals $\sigma_{0}$ at a critical distance $d=L$. Figure 1 shows a comparison of the predictions using the Point Method, the Line Method and the Notch Effective Stress for a number of materials and notches reported in the literature.

\section{Conclusions}

A new effective stress expression and its critical distance directly obtained for notches is proposed. The Notch Effective Stress (NES) is expressed as the geometric mean of the mean stress and the local stress evaluated the critical distance of the material proposed by Taylor. Its applicability is compared successfully with the LM and PM predictions, which were originally established for cracks.

\section{References}

[1] D. Taylor, Int J Fatigue 21, 413-20 (1999)

[2] D. Taylor, Elsevier Science, Oxford, UK (2007)

[3] D. Taylor, Eng. Fail. Anal. 12, 6 (2010)

[4] C. Vallellano, PhD Thesis, University. of Sevilla (1998)

[5] C. Vallellano, A. Navarro, J. Dominguez, Phil. Mag. A 82, 1 (2002) 